\title{
Liquid argon scintillation detection utilizing wavelength-shifting plates and light guides
}

\author{
B. Howard on behalf of DUNE collaboration" \\ Indiana University, \\ Bloomington, IN, USA \\ E-mail: bruhowar@indiana.edu
}

\begin{abstract}
In DUNE, the event timing provided by the detection of the relatively prompt scintillation photons will improve spatial resolution in the drift direction of the time-projection chamber (TPC) and is especially useful for non-beam physics topics such as supernova neutrinos and nucleon decay. The baseline design for the first 10kt single phase TPC fits the photon detector system in the natural gap between the wire planes of adjacent TPC volumes. A prototype photon detector design utilizes wavelength-shifter coated plates to convert the vacuum ultraviolet scintillation light to the optical and commercially-produced wavelength-shifting light guides to trap some of this light and transport it to an array of silicon photomultipliers at the end. This system and the testing performed to characterize the system and determine the efficiency are discussed.
\end{abstract}

KeYwords: Noble liquid detectors (scintillation, ionization, double-phase); Photon detectors for UV, visible and IR photons (solid-state) (PIN diodes, APDs, Si-PMTs, G-APDs, CCDs, EBCCDs, EMCCDs etc); Neutrino detectors

This document was prepared by DUNE collaboration using the resources of the Fermi National Accelerator Laboratory (Fermilab), a U.S. Department of Energy, Office of Science, HEP User Facility. Fermilab is managed by Fermi Research Alliance, LLC (FRA), acting under Contract No. DE-AC02-07CH11359.

${ }^{0}$ FERMILAB-CONF-17-597-ND-PPD 


\section{Contents}

1 Introduction and description of technology 1

2 Characterization studies 2

2.1 Component testing in the laboratory 2

2.2 Prototype test in LAr 3

3 Discussion 5

\section{Introduction and description of technology}

The Deep Underground Neutrino Experiment (DUNE) is a next-generation neutrino experiment which will place a detector nearly a mile underground in Lead, South Dakota. This detector will be comprised of four 10kt fiducial volume liquid argon (LAr) time-projection chambers (TPCs) and will study neutrino oscillations via a neutrino beam from Fermilab. The large and sensitive detectors enable non-beam physics studies as well, for example nucleon decay and supernova neutrinos.

While the main detector system is the TPC sensitive to the ionization signal from the propagation of charged particles, LAr also yields strong scintillation in the vacuum ultraviolet (VUV) at $128 \mathrm{~nm}$. The scintillation photons provide a prompt signal in coincidence with events, and together with the ionization signal arrival times, this is capable of providing $\sim \mathrm{mm}$ resolution in the drift direction for the TPC [1]. This is especially useful for non-beam physics, for which no expected event time is known. For the single-phase detector design, the cathodes and anodes are both submerged in LAr. In the baseline design for the first single-phase DUNE detector, the photon detection (PD) system is intended to fit within the anode plane assemblies (APAs) - outside the fiducial volume - between sets of TPC wires.

Due to the difficulty of detecting VUV photons, especially over such large areas, wavelength shifing solutions are typically employed. A prototype PD module in development for large LAr TPCs like DUNE utilizes acrylic plates coated with the wavelength-shifter tetraphenyl-butadiene (TPB) placed in front of and behind wavelength-shifting polystyrene light guides from Eljen Technology ${ }^{1}$. The plates are sprayed with a solution containing TPB and heated to embed the wavelength-shifter in the outer layer. This configuration is sensitive to events on both sides of a given APA, especially useful for the central APA planes, where both sides are separate TPC volumes.

A depiction of this prototype PD system technology is shown in Figure 1. The dimensions shown are the approximate dimensions for the module tested in characterization studies, though full-scale modules would be approximately $2.1 \mathrm{~m}$ in length. The plates are responsible for the initial conversion, with the measured emission spectrum of samples peaking around $430 \mathrm{~nm}$. Some of the converted light will then fall onto the Eljen light guide, which contains the wavelength-shifter EJ280. The EJ-280 absorption spectrum is well-matched to the emission spectrum, as shown in Figure

\footnotetext{
${ }^{1}$ http://www.eljentechnology.com/
} 
2 (left). The EJ-280 emission spectrum peaks a bit below 500nm. The commercially-produced light guide bar then traps photons via total internal reflection at the polystyrene-LAr interface and transports them to an array of silicon photomultipliers (SiPMs) at one end. For the prototype discussed here, SensL ${ }^{2}$ SiPMs recorded signals reaching the readout end. There is good overlap of the SiPM photon detection efficiency (PDE) and the EJ-280 emission, as shown in Figure 2 (right).

While this PD system requires two wavelength shifts, it decouples the conversion process from the transport process. Thus, one is more free to optimize the wavelength-shifting plates without degrading the light guide performance. Further, the commercial production of the light guides allows for better quality assurance, quality control, and overall reliability.

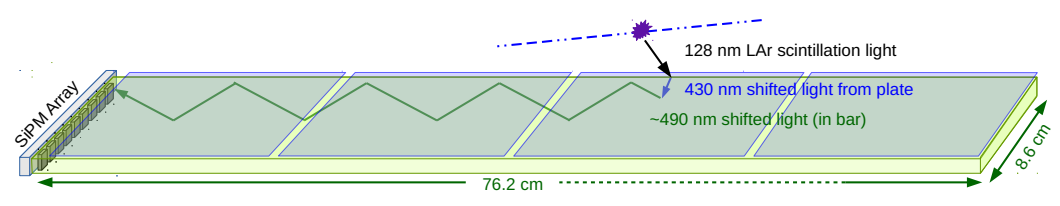

Figure 1. Depiction of prototype photon detection system showing the processes involved in converting the VUV scintillation into detected signal. Full-scale modules would be approximately $2.1 \mathrm{~m}$ in length.
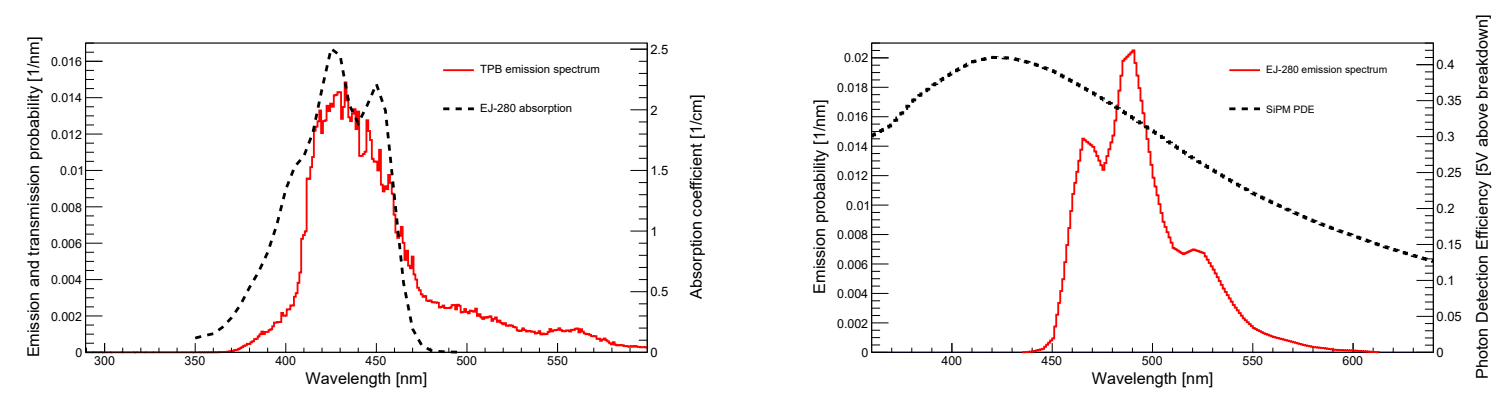

Figure 2. (left) TPB emission (red) measured with plate samples and the absorption of EJ-280 (black) from Eljen. (right) EJ-280 emission (red) from Eljen and the detection probability of SiPMs (black) from SensL.

\section{Characterization studies}

\subsection{Component testing in the laboratory}

The laboratory tests performed on the individual components comprising the system and the integrated prototype test described in the next section are described in detail elsewhere [2]. One example is a study of the attenuation effects of the light guide conducted by producing scintillation signals with an $\alpha$ source and wavelength-shifting plate sample traveling along the light guide.

Further, a VUV monochromator was used to test the efficiency of samples from the wavelengthshifting plates. Using the monochromator to select an output wavelength from a deuterium lamp, the current read out by an SiPM placed behind an exposed plate sample is compared to the current read out by a VUV-sensitive photodiode when no plate is present. For relative efficiency determinations, comparing the output to incident light in this way is sufficient. However, to determine the absolute

\footnotetext{
${ }^{2} \mathrm{http}: / / \mathrm{sensl} . \mathrm{com} /$
} 
efficiency of these plates, one needs to further consider the responsivity [A/W] of the photodiode and SiPM, geometrical corrections for the size of the beam at the detector, and transmission of converted light through the acrylic. The resulting plate efficiencies were determined to range from $\sim 49 \%$ to $\sim 66 \%$ with fractional uncertainties estimated to be a bit over $10 \%$. Three of the four plates were determined to be $>60 \%$. With commercialization and quality control, it is believed that the final products could be more uniform and consistent.

The laboratory measurements of components and information from the manufacturers are used in a custom Monte Carlo simulation to predict the efficiency of the prototype PD system to VUV scintillation photons incident on the module. It starts with photons generated at a random location on the surface of a plate, with direction picked from a Lambertian (cosine) distribution [3] from the normal to the plate surface. The simulation tracks photons, determining if they will undergo various processes, such as hitting the light guide, being absorbed by the EJ-280, being re-emitted, being trapped by total internal reflection, being re-absorbed/re-emitted by the EJ-280, reaching the readout, and being detected by SiPMs. Any of these processes can result in the loss of photons. An additional destruction probability checked at each reflection is used to characterize otherwise unaccounted losses of photons. This probability was chosen for the light guide to give a good match between the attenuation measurement described above and a version of this simulation approximating the conditions of that scan (see Figure 3). In simulations run to predict module efficiency, weights correct for the SiPM coverage and plate efficiencies.

In Figure 5, at the end of Section 2.2, the results of this simulation are shown for a module with each plate efficiency set equal to the exposure-weighted average of the four individual efficiencies in the prototype test in LAr. This simulation also produces a curve like the red line in Figure 3, but due to the different setup, the curve will be a somewhat different shape. A double exponential fit to this result gives the transport function describing attenuation effects

$$
\text { transport }=0.29 e^{-x / 4.3 \mathrm{~cm}}+0.71 e^{-x / 225 \mathrm{~cm}}
$$

where the coefficients are normalized to 1 at the readout end $(\mathrm{x}=0)$. If the plate efficiency used is the strict average of the 3 brighter plates, the simulation yields a predicted efficiency of $0.48 \%$ at the readout end when fit with the transport function and a free scale parameter, that is

$$
\epsilon=0.0048\left(0.29 e^{-x / 4.3 \mathrm{~cm}}+0.71 e^{-x / 225 \mathrm{~cm}}\right)
$$

Averaging $\epsilon$ over $2.1 \mathrm{~m}$ to approximate uniform illumination yields $\sim 0.22 \%$ efficiency.

\subsection{Prototype test in LAr}

The Fermilab LAr testing facilities provide large dewars ( 100s of liters) for testing. Filtration on the input line removes much of the $\mathrm{O}_{2}$ and $\mathrm{H}_{2} \mathrm{O}$ contamination as the dewar fills, and contamination monitors allow further monitoring. Further, a condenser helps maintain LAr levels. The $\sim 76 \mathrm{~cm}$ long prototype with four prototype wavelength-shifting plates discussed above was exposed to cosmic-ray muons in the Blanche dewar facility at Fermilab.

A four-fold coincidence trigger, based on signals from a hodoscope borrowed from the CREST cosmic ray experiment and additional scintillator paddles, was used to trigger on cosmic-ray muons. Through-going muon tracks are selected by requiring only one PMT firing on each hodoscope, and 


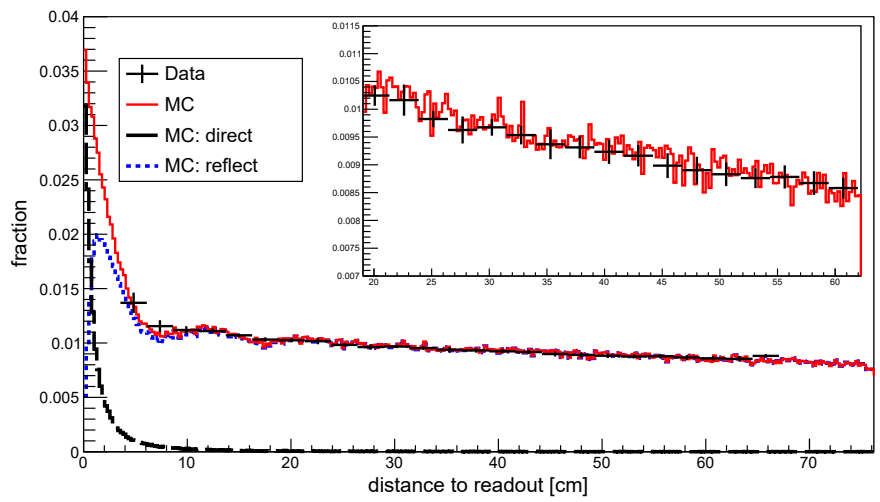

Figure 3. The results of the attenuation study are shown by the black crosses, normalized to the area under the same region in the simulation. The simulation (red curve) approximates the experiment conducted and determines the number of photons detected per photon initially at a location in the light guide. It is used to determine a reasonable loss probability at reflections for the simulations. The blue dots and black dashes break the simulation result into the light which has undergone some or no reflections, respectively.

further cuts are applied to largely reject other backgrounds. The waveforms from the $8 \mathrm{SiPMs}$ used with the prototype were summed to yield the number of collected photoelectrons (PE), $N_{\text {det }}$.

A Monte Carlo simulation was developed to throw photons from a given track trajectory. The muons are assumed to deposit minimum ionization energy in LAr [4], and it is assumed that MIPs should deposit $~ 40,000$ photons/MeV [5]. This simulation tracks photons through an approximation of the dewar geometry by ray-tracing and includes processes for reflection, absorption, and scattering. The result is an estimate for the number and locations of scintillation photons falling on the light guide from a given track, which is corrected for attenuation by applying the transport function in Section 2.1 bin by bin. This provides the number of photons expected at the readout end if the module was perfectly efficient in every other process $\left(N_{\text {exp }}\right)$. In this sense, the ratio of PE digitized per expected photon at the readout end is a proxy for module efficiency at the readout end. The resulting distribution for all analyzed tracks is fit with the sum of an exponential to handle the background and a Landau to characterize the response to muon tracks. To deal with estimated errors in the ratio, a number of histograms are made in which the ratio of $\frac{N_{\text {det }}}{N_{\exp }}$ is allowed to vary by randomly choosing from a Gaussian. The average ratio histogram is depicted in Figure 4 and is overlayed by the function with the average fit parameters. To give a sense for the spread in values bin by bin in the histogram, the standard deviation of bin contents is shown in Figure 4 as well. The average most probable value from the fits is $\frac{N_{\text {det }}}{N_{\exp }}=0.0035$. To put this in terms of the 12 SiPMs that can be arrayed across the end of the light guides and used in the simulations, this value is corrected by the factor 12/8. Further, following the arguments in [6], to convert the efficiency from [PE/photon in] into [photons/photon in], a correction factor of $\sim 1.26^{-1}$ is needed to account for the PE/photon ratio due to cross-talk. This results in a module efficiency of $0.41 \%$ at the readout end. To characterize this as a function of distance from the readout end, this is multiplied by the transport function in Section 2.1.

The comparison of the simulated result using the weighted average expectation for photon illumination in Blanche can then be compared to the result of the analysis of through-going cosmic 
ray tracks in Blanche. This comparison appears in Figure 5, and shows reasonable agreement between the two characterizations. This suggests the results are consistent with 40,000 photons $/ \mathrm{MeV}$ for approximately minimum ionizing particles [5].

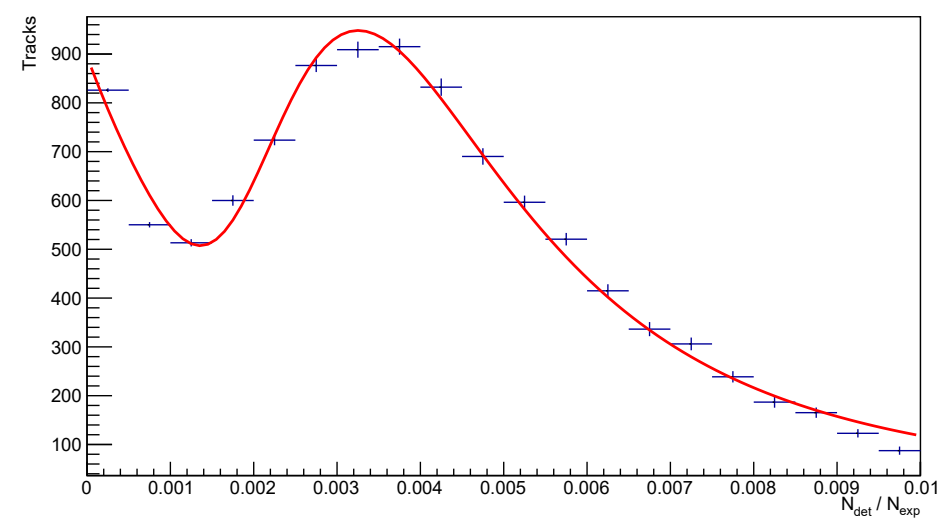

Figure 4. Average histogram of $\frac{N_{\text {det }}}{N_{\text {exp }}}$, where the standard deviation of the results are shown for each bin to characterize the spread. The average of the fit functions characterizing the module efficiency is shown in red.

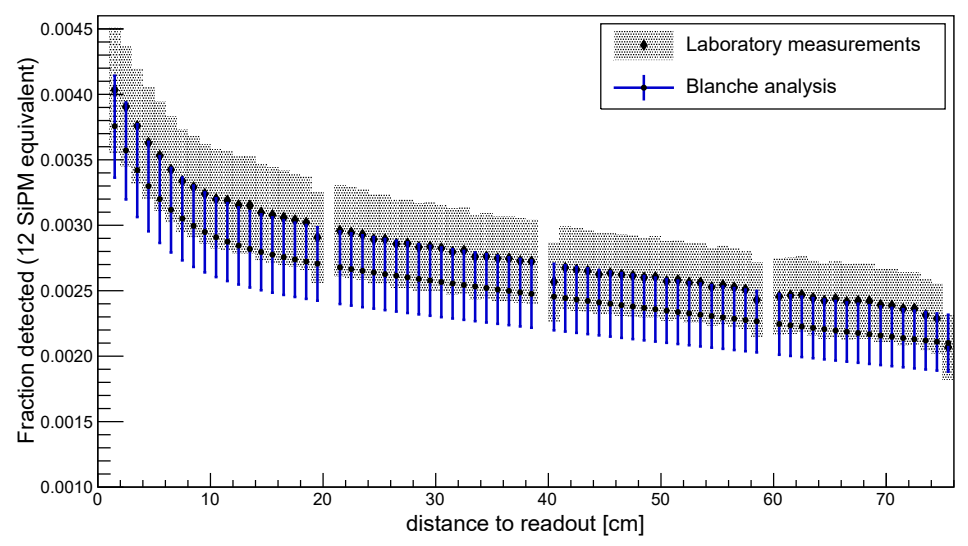

Figure 5. The gray curve shows the predicted efficiency from the simulation described in Section 2.1 with plate efficiencies set to the weighted average for the exposure in Blanche. The blue curve is the prototype test result: the efficiency at the readout multiplied by the transport function.

\section{Discussion}

This proceeding details a prototype scintillation photon detection system using wavelength-shifting plates and commercial light guides for use in large LAr TPCs like DUNE. A set of tests to characterize the detectors and a prototype test in LAr at Fermilab were discussed. Prototypes will be tested in the upcoming protoDUNE-SP experiment at CERN [7], and it is believed that there is still significant room for improvement on the prototype design discussed here. For example, it is expected that one could increase efficiency while reducing channel count with double-ended readout and ganged SiPMs. 


\section{Acknowledgments}

Special thanks to the other coauthors of the paper detailing the characterization and prototype studies. They are: S. Mufson (IU), D. Whittington (IU/Syracuse), B. Adams (IU), B. Baugh (IU), J.R. Jordan (IU/Michigan), J. Karty (IU), C.T. Macias (IU), and A. Pla-Dalmau (Fermilab). Thanks additionally to those who helped make these studies possible. At IU: M. Gebhard, M. Lang, B. Martin, J. Musser, P. Smith, J. Urheim. At Fermilab: R. Davis, C. Escobar, B. Miner, E. Niner, S. Pordes, B. Rebel, F. Schwartz, M. Zuckerbrot. At Argonne National Lab: J. Anderson, G. Drake, A. Kreps, M. Oberling. At Colorado State: N. Buchanan, J. Jablonski, D. Warner. At Eljen Technology: C. Hurlbut. Finally, thanks to the many other individuals working on developing the photon detection system for DUNE.

This work was supported in part by the Trustees of Indiana University, the DOE Office of High Energy Physics through grant DE-SC0010120 to Indiana University, and grant \#240296 from Broookhaven National Laboratory to Indiana University.

This manuscript has been authored by Fermi Research Alliance, LLC under Contract No. DE-AC02-07CH11359 with the U.S. Department of Energy, Office of Science, Office of High Energy Physics. The United States Government retains and the publisher, by accepting the article for publication, acknowledges that the United States Government retains a non-exclusive, paid-up, irrevocable, world-wide license to publish or reproduce the published form of this manuscript, or allow others to do so, for United States Government purposes.

\section{References}

[1] R. Acciarri et al., Long-Baseline Neutrino Facility (LBNF) and Deep Underground Neutrino Experiment (DUNE) Conceptual Design Report, Volume 4: The DUNE Detectors at LBNF, arxiv:1601.02984 [physics.ins-det].

[2] B. Howard et al., A Novel Use of Light Guides and Wavelength Shifting Plates for the Detection of Scintillation Photons in Large Liquid Argon Detectors, arxiv: 1710.11233 [physics.ins-det].

[3] V. Gehman et al., Fluorescence efficiency and visible re-emission spectrum of tetraphenyl butadiene films at extreme ultraviolet wavelengths, NIM A654 (2011) 116.

[4] C. Patrignani et al., The Review of Particle Physics, Chin. Phys. C 40 (2017) 10001.

[5] T. Doke et al., Estimation of absolute photon yields in liquid argon and xenon for relativistic ( $1 \mathrm{mev})$ electrons, NIM A291 (1990) 617.

[6] L. Gallego et al., Modeling crosstalk in silicon photomultipliers, JINST 5 (2013) P05010.

[7] B. Abi et al., The Single-Phase ProtoDUNE Technical Design Report, arxiv:1706.07081 [physics.ins-det]. 